\title{
Comparison of Different Treatment Modalities for Type 1 Diabetes, Including Sensor-Augmented Insulin Regimens, in 52 Weeks of Follow-Up: A COMISAIR Study
}

\author{
Jan Šoupal, MD, Lenka Petruželková, MD, Milan Flekač, MD, PhD, Tomáš Pelcl, MD, \\ Martin Matoulek, MD, PhD, Martina Daňková, MSc, Jan Škrha, MD, DrSc, MBA, \\ Štěpán Svačina, MD, DrSc, MBA, and Martin Prázný, MD, $\mathrm{PhD}^{1}$
}

\begin{abstract}
Objective: To compare different treatment modalities for patients with type 1 diabetes (T1D) based on real-time continuous glucose monitoring (RT-CGM) or self-monitoring of blood glucose (SMBG) combined with multiple daily injections (MDIs) or continuous subcutaneous insulin infusion (CSII).

Research Design and Methods: Sixty-five T1D patients were followed up for a year. Of these, 27 started RTCGM as part of a sensor-augmented insulin regimen (SAIR); within this SAIR group, 15 subjects started sensoraugmented pump (SAP) therapy and the remaining 12 continued with MDIs (MDIs + RT-CGM). A second group of 20 patients initiated CSII without RT-CGM, while a third group of 18 subjects continued on MDIs and SMBG. The main endpoints were reduction of $\mathrm{HbA}_{1 \mathrm{c}}$, glycemic variability $(\mathrm{GV})$, and incidence of hypoglycemia.

Results: After a year, the baseline mean $\mathrm{HbA}_{1 \mathrm{c}}$ in the SAIR group $(8.3 \%)$ decreased to $7.1 \%(P<0.0001)$; both SAIR subgroups, SAP and MDIs + RT-CGM, showed comparable improvement. The CSII group also had reduced $\mathrm{HbA}_{1 \mathrm{c}}(8.4 \% \pm 0.9 \%$ vs. $7.9 \% \pm 0.7 \% ; P<0.05)$. Both SAIRs were superior to MDIs $(P=0.002)$ and CSII $(P=0.0032)$. GV was also lowered, both in the SAIR $(P<0.0001)$ and CSII $(P<0.05)$ groups. Reduced incidence of hypoglycemia was observed only with SAIR $(8 \% \pm 4 \%$ vs. $6 \% \pm 3 \% ; P<0.01)$.

Conclusion: Both SAIRs, SAP and MDIs + RT-CGM, provided significant and comparable decrease of $\mathrm{HbA}_{1 \mathrm{c}}$ with concurrent reduction of hypoglycemia. This improvement was greater than that seen with CSII. The combination of RT-CGM and MDIs can be a suitable alternative to SAP for some patients.
\end{abstract}

\section{Introduction}

T HERE HAVE BEEN many advances in diabetes care technologies in the last few years, which have resulted in new opportunities for diabetes treatment. Despite some encouraging results, metabolic control remains suboptimal in most patients with type 1 diabetes. ${ }^{1}$

Successful treatment of type 1 diabetes requires both a precise insulin delivery system and reliable glucose monitor- ing systems. For delivery systems, the two most common are multiple daily injection (MDI) and continuous subcutaneous insulin infusion (CSII) therapies. ${ }^{2,3}$ With both strategies, bolus insulin is dosed based on several factors, including carbohydrate content and glycemia. There are also two common monitoring systems: classical self-monitoring of blood glucose (SMBG) and real-time continuous glucose monitoring (RTCGM). With SMBG, even if frequent monitoring is performed, some potentially important trends are always missed because

\footnotetext{
${ }^{1}$ 3rd Department of Internal Medicine, 1st Faculty of Medicine, Charles University in Prague, Czech Republic.

${ }^{2}$ Department of Paediatrics, 2nd Faculty of Medicine, Charles University in Prague, Czech Republic.

(c) Jan Šoupal, et al., 2016; Published by Mary Ann Liebert, Inc. This Open Access article is distributed under the terms of the Creative Commons Attribution Noncommercial License (http://creativecommons.org/licenses/by-nc/4.0/) which permits any noncommercial use, distribution, and reproduction in any medium, provided the original author(s) and the source are credited.
} 
they occurred between two measurements. In contrast, CGM gives the concentration of glucose in subcutaneous tissue approximately every $5 \mathrm{~min}$ and therefore provides much more data, ${ }^{4}$ including glucose trends, to which patients can react to prevent hyper- and/or hypoglycemia.

Despite limited data, it is commonly believed that optimal diabetes management can best be achieved when an RTCGM is used in combination with insulin pump therapysensor-augmented pump (SAP) therapy. ${ }^{5}$ It has been reported that SAP improves glycemic control, ${ }^{6,7}$ reduces time spent in hypoglycemia, increases time spent in the target zone,,${ }^{7,8}$ and decreases glycemic variability $(\mathrm{GV}){ }^{9,10}$

In contrast, the efficacy of the combination of real-time CGM with MDIs is less described. Moreover, the accuracy and usability of CGM have gradually improved. Therefore, we need data from clinical studies with the newer generation of CGM devices. Finally, prospective studies simultaneously comparing head-to-head the different combinations of insulin delivery and monitoring systems-MDIs + SMBG, MDIs + RT-CGM, CSII + SMBG, and SAP-are lacking. Such a study would help to elucidate whether the observed benefit of SAP use is secondary to the RT-CGM technology, the type of insulin delivery, or both.

The aim of the study was to compare the efficacy of longterm use of sensor-augmented insulin regimens (SAIRs), that is, RT-CGM combined with either CSII or MDIs, on glycemic control compared with more common schemes based on classical SMBG.

\section{Research Design and Methods}

\section{Study population}

Sixty-five patients with type 1 diabetes were enrolled at the 3rd Department of Internal Medicine, 1st Faculty of Medicine, Charles University in Prague, Czech Republic. All subjects provided written informed consent before enrollment. Participants were included if they were aged $>18$ years, had a duration of diabetes of more than 2 years, and had an $\mathrm{HbA}_{1 \mathrm{c}}$ level between $7.0 \%$ and $10 \%(53$ and $86 \mathrm{mmol} / \mathrm{mol})$. Only patients with insulin analogs were enrolled in this study. Subjects who had used CGM during the past 3 months were excluded from the study. Patients with ketoacidosis within the past 3 months and/or severe noncompliance and/or any concomitant therapy influencing glucose metabolism, pregnant women, and women planning pregnancy were not allowed to participate either. Patients were divided into three groups with comparable baseline parameters (Table 1), taking into account their preferences and diabetologist's recommendation. At the baseline, 27 patients started to use RT-CGM as part of an SAIR, 20 patients initiated CSII therapy (without RT-CGM), and 18 patients continued on MDIs and SMBG only.

In the SAIR group, after a further consultation with the diabetologist, subjects could choose a combination of RTCGM with either an insulin pump (SAP) or MDIs. Fifteen of them started to use SAP and the remaining 12 continued with MDIs (MDIs + RT-CGM).

A prerequisite for participation in the SAIR group was the willingness to use sensors $>70 \%$ of the time. Similarly, patients in the groups without CGM had to be willing to monitor their glucose (SMBG) at least four times a day.

\section{Study procedures}

This was a nonrandomized, prospective, real-life clinical trial. Subjects were scheduled for a total of seven clinic visits (initial, at 2 weeks, 1 month, then 3, 6, 9, and 12 months). Initially, all patients were monitored by professional CGM (iPro2 ${ }^{\mathrm{TM}}$; Medtronic, Northridge, CA) for 6 days. Throughout the study, subjects in the groups not using SAIR had professional CGM every 3 months. Participants in the CSII group wore one of two types of insulin pumps, MiniMed Paradigm Veo (Medtronic) and Animas Vibe (Animas Corporation, West Chester, PA). Participants in the SAP subgroup used either the MiniMed Paradigm Veo System with Enlite sensors (Medtronic) or Animas Vibe system with DexCom G4 sensors (Dexcom, San Diego, CA). The subgroup of patients with MDIs + RT-CGM used a DexCom G4 CGM system comprising a 7-day transcutaneous sensor, a transmitter, and a receiver. The patients were provided with a personal blood glucose meter (OneTouch [LifeScan, Milpitas, CA] or CONTOUR $^{\mathrm{TM}}$ LINK [Bayer Diabetes Care, Basel, Switzerland]), which was used for diabetes self-management purposes and calibration of CGM. At the baseline, all subjects underwent a structured 4-day training program. In the first part of this program, specialists reviewed general principles of type 1 diabetes management. Patients were educated on how to prevent hypoglycemia and deal with it in a variety of situations. They

Table 1. Baseline Characteristic of Patients

\begin{tabular}{lccccc}
\hline & \multicolumn{3}{c}{ SAIR group } & CSII + SMBG & MDIs + SMBG \\
\cline { 2 - 4 } Characteristic & All & SAP therapy & MDIs + CGM & group & group \\
\hline No. & 27 & 15 & 12 & 20 & 18 \\
Male [n (\%)] & $16(59)$ & $9(60)$ & $7(58)$ & $11(55)$ & $9(50)$ \\
Age (years) & $34 \pm 10$ & $33 \pm 10$ & $34 \pm 10$ & $35 \pm 9$ & $38 \pm 17$ \\
Duration of diabetes (years) & $15 \pm 9$ & $15 \pm 9$ & $16 \pm 10$ & $13 \pm 10$ & $14 \pm 9$ \\
HbA $_{1 \mathrm{c}}(\mathrm{mmol} / \mathrm{mol})$ & $67.5 \pm 10$ & $66 \pm 9$ & $69.3 \pm 8$ & $67.9 \pm 9$ & $67.2 \pm 9$ \\
$\mathrm{HbA}_{1 \mathrm{c}}(\%)$ & $8.3 \pm 9$ & $8.2 \pm 0.9$ & $8.5 \pm 1.1$ & $8.4 \pm 0.8$ & $8.3 \pm 0.8$ \\
Mean sensor glucose (mmol/L) & $10.7 \pm 1.5$ & $10.4 \pm 1.4$ & $10.9 \pm 1.6$ & $10.7 \pm 1.2$ & $10.6 \pm 1.4$ \\
BMI $\left(\mathrm{kg} / \mathrm{m}^{2}\right)$ & $25 \pm 3$ & $25 \pm 3$ & $25 \pm 3$ & $26 \pm 4$ & $24 \pm 3$ \\
\hline
\end{tabular}

Values are presented as mean $\pm \mathrm{SD}, P$ values $<0.05$ are statistically significant.

CGM, continuous glucose monitoring; CSII, continuous subcutaneous insulin infusion; MDIs, multiple daily injections; SAIR, sensoraugmented insulin regimen; SAP, sensor-augmented pump; SMBG, self-monitoring of blood glucose. 
were informed about the appropriate timing of preprandial insulin dosing. All patients underwent theoretical and practical education in carbohydrate counting and were encouraged to use flexible dosing of insulin throughout this study. Only patients in the SAIR and CSII groups completed theoretical training on the relevant devices, followed by treatment initiation and practical training with investigators.

Participants on SAIR were encouraged to make selfadjustments to their treatment using CGM values, hyper- and hypoglycemic alerts and trends, and also to incorporate results of SMBG into treatment changes. The target range for glucose was usually initially relatively wide, but we emphasized to patients that its successive narrowing is usually necessary for reduction of mean blood glucose and GV. An important part of the training was management of problems with CGM (troubleshooting) related to sweating, skin reactions, alarm settings, and appropriate calibration according to the type of CGM system. We highlighted to patients the importance of regular downloading and review of the data from CGM devices and insulin pumps. A bolus calculator was set for all patients with insulin pumps. Subjects in non-SAIR groups were encouraged to measure their blood glucose at least four times a day. All patients were instructed to use only the study blood glucose meter provided to them for all SMBG measurements taken during this trial. Data from all CGM systems, insulin pumps, and blood glucose meters were downloaded for analysis.

\section{Prespecified outcomes}

The primary endpoint was the difference in $\mathrm{HbA}_{1 \mathrm{c}}$ between the groups after 52 weeks of follow-up. $\mathrm{HbA}_{1 \mathrm{c}}$ values were measured at the baseline, then every 3 months, and at the end of this trial. $\mathrm{HbA}_{1 \mathrm{c}}$ was analyzed by a high-performance liquid chromatography method on a Variant II analyzer (BioRad, Hercules, CA). The normal reference range of $\mathrm{HbA}_{1 \mathrm{c}}$ in our laboratory is $4.0 \%-6.0 \%(20-42 \mathrm{mmol} / \mathrm{mol})$.

Prespecified secondary endpoints were changes of GV expressed by the total standard deviation of blood glucose $\left(\mathrm{SD}_{T}\right)$, average daily glucose from CGM, \% of time spent in range (4.0-10.0 $\mathrm{mmol} / \mathrm{L})$, and the incidence of hypoglycemia (\% of time below $3.9 \mathrm{mmol} / \mathrm{L}$ ).

At each clinic visit, patients were screened for adverse events and sensor insertion sites were inspected. Severe hypoglycemia was defined as an episode requiring assistance from another person or neurological recovery in response to restoration of plasma glucose to normal. Ketoacidosis was defined as an episode of hyperglycemia $(>14 \mathrm{mmol} / \mathrm{L})$ with low serum bicarbonate $(<15 \mathrm{mmol} / \mathrm{L})$, low $\mathrm{pH}(<7.3)$, or both

Table 2. Insulin Treatment Patterns, Self-monitoring of Blood Glucose, and Body Weight at The Baseline and AT The END of The Study

\begin{tabular}{|c|c|c|c|c|}
\hline & At the baseline & At the end & $\mathrm{P}$ & $95 \% C I$ \\
\hline \multicolumn{5}{|c|}{ No. of boluses/day $(n)$} \\
\hline SAIR group & $4.0 \pm 0.7$ & $6.8 \pm 2.2$ & $<0.0001^{*}$ & 1.8679 to 3.6860 \\
\hline SAP & $4.0 \pm 0.8$ & $7.2 \pm 2.3$ & $<0.0001^{*}$ & 1.9379 to 4.6335 \\
\hline MDI + CGM & $4.0 \pm 0.5$ & $6.2 \pm 2$ & 0.002 & 0.9308 to 3.4359 \\
\hline CSII & $4.1 \pm 0.8$ & $4.7 \pm 1.4$ & 0.08 & -0.08277 to 1.3628 \\
\hline MDIs & $3.9 \pm 0.8$ & $3.9 \pm 0.8$ & 0.83 & -0.6024 to 0.4847 \\
\hline \multicolumn{5}{|c|}{ Relative proportion of bolus insulin (\%) } \\
\hline SAIR group & $49 \pm 7$ & $53 \pm 5$ & $0.004 *$ & 1.5371 to 7.8200 \\
\hline SAP & $49 \pm 7$ & $54 \pm 4$ & $0.03 *$ & 0.3877 to 9.4790 \\
\hline MDI + CGM & $48 \pm 6$ & $53 \pm 5$ & 0.07 & -0.3165 to 9.08588 \\
\hline CSII & $50 \pm 9$ & $52 \pm 7$ & 0.38 & -2.8715 to 7.3715 \\
\hline MDIs & $50 \pm 5$ & $52 \pm 6$ & 0.45 & -2.3613 to 5.2502 \\
\hline \multicolumn{5}{|c|}{ The total daily dose of insulin (U) } \\
\hline SAIR group & $46 \pm 12$ & $48 \pm 13$ & 0.58 & -4.9325 to 8.7843 \\
\hline SAP & $45 \pm 12$ & $47 \pm 13$ & 0.65 & -7.2315 to 11.3648 \\
\hline MDIs + CGM & $48 \pm 12$ & $50 \pm 13$ & 0.75 & -9.3076 to 12.8076 \\
\hline CSII & $48 \pm 13$ & $47 \pm 13$ & 0.98 & -8.2209 to 8.0209 \\
\hline MDIs & $47 \pm 14$ & $48 \pm 14$ & 0.85 & -8.5492 to 10.3270 \\
\hline \multicolumn{5}{|c|}{ Frequency of SMBG/day $(n)$} \\
\hline SAIR group & $3.8 \pm 1.2$ & $3.2 \pm 1.0$ & $0.049 *$ & -1.1759 to -0.0019 \\
\hline SAP & $3.7 \pm 0.8$ & $3.6 \pm 1.0$ & 0.84 & -0.7408 to 0.6074 \\
\hline MDIs + CGM & $3.9 \pm 1.6$ & $2.7 \pm 0.6$ & $0.02^{*}$ & -2.2431 to -0.2402 \\
\hline CSII & $3.6 \pm 0.7$ & $3.6 \pm 0.7$ & 0.95 & -0.5001 to 0.4686 \\
\hline MDIs & $3.6 \pm 1.3$ & $3.7 \pm 1.4$ & 0.88 & -0.8935 to 1.0346 \\
\hline \multicolumn{5}{|l|}{ Body weight $(\mathrm{kg})$} \\
\hline SAIR group & $77.7 \pm 11$ & $79.0 \pm 12$ & 0.68 & -4.9653 to 7.5208 \\
\hline SAP & $76.1 \pm 10$ & $77.3 \pm 9$ & 0.74 & -5.8359 to 8.1692 \\
\hline MDIs + CGM & $79.6 \pm 13$ & $81.0 \pm 14$ & 0.80 & -10.2345 to 13.0679 \\
\hline CSII & $74.1 \pm 12$ & $74.4 \pm 12$ & 0.94 & -7.3909 to 7.9909 \\
\hline MDIs & $73 \pm 13$ & $73.5 \pm 14$ & 0.92 & -8.7031 to 9.6698 \\
\hline
\end{tabular}

Values are presented as mean $\pm \mathrm{SD} ; * P$ values $<0.05$ are statistically significant.

CI, confidence interval. 
together with either ketonemia or ketonuria that required treatment in a healthcare facility.

\section{Statistical analysis}

Statistical evaluation was performed by Statistics for Windows version 10 software (SPSS, Inc., Chicago, IL). Basic descriptive statistics were calculated for the relevant parameters. Analysis was performed by nonparametric tests (Kruskal-Wallis, Wilcoxon, and ANOVA repeated measurement). Data are expressed as mean-SD values. A value of $P<0.05$ was considered statistically significant.

\section{Results}

\section{Baseline characteristics and adherence}

Baseline characteristics were similar in the three groups (Table 1). Of the 65 patients enrolled, 62 completed all study visits. One subject from the CSII group and one from the SAIR group withdrew from the study after the third visit because of personal reasons. One patient from the MDI group was excluded from the analysis due to significant protocol violation. Mean sensor percentage use in the SAIR group was $85 \% \pm 10 \%$ of the time (median $85 \%$ ) with no significant differences between the two subgroups-SAP or MDIs + RTCGM $(85 \% \pm 10 \%$ [median $84 \%$ ] vs. $85 \% \pm 10 \%$ [median 87\%]; $P=0.98)$.

Compared with the baseline, at the end of this study in the SAIR group, there was a significantly higher number of boluses per day and the relative proportion of bolus insulin was higher, while no significant change in these parameters was seen in either SMBG group (Table 2). No change in the total daily dose of insulin between the baseline and the end of the study was observed for any study group (Table 2 ).

The average number of boluses per day at the end of the study was lower in both SMBG groups in comparison with the SAIR group $(6.8 \pm 2.2$ vs. $4.3 \pm 1.2 ; P<0.0001)$. A higher frequency of boluses was seen in patients with CSII versus the self-reported boluses in the MDI only group ( $4.7 \pm 1.4 \mathrm{vs}$. $3.9 \pm 0.8 ; P=0.04)$, while no significant difference between SAP and MDIs + RT-CGM was observed (7.2 \pm 2.3 vs. $6.2 \pm 2 ; P=0.25)$. At the end of this trial, the total daily dose of insulin and the relative proportion of bolus insulin were not different between study groups (Table 2).

No significant change in body weight between the beginning and the end of the study was found for any study group (Table 2).

At the end of this study, the average number of blood glucose tests in non-SAIR groups was $3.7 \pm 1.1$ per day (median 3.6/day), with no significant differences between the groups with MDIs and CSII (3.7 \pm 1.4 [median 3.3/day] vs. $3.6 \pm 0.7$ [median 3.5/day]; $P=0.8$ ). In comparison with SMBG groups, the average frequency of finger-stick tests performed per day was numerically, but not statistically, lower in the SAIR group $(3.2 \pm 1.0$ [median 3.1/day] vs. $3.7 \pm 1.1$ [median 3.6/day]; $P=0.08$ ). However, regardless of the type of insulin delivery (SAP or MDIs + RT-CGM), there was lower frequency of SMBG in subjects who were using the DexCom G4 sensor $(n=19)$ in comparison with users of the MiniMed Paradigm Veo System with Enlite sensors $(n=8)(2.7 \pm 0.6$ vs. $4.3 \pm 0.7, P<0.001)$.

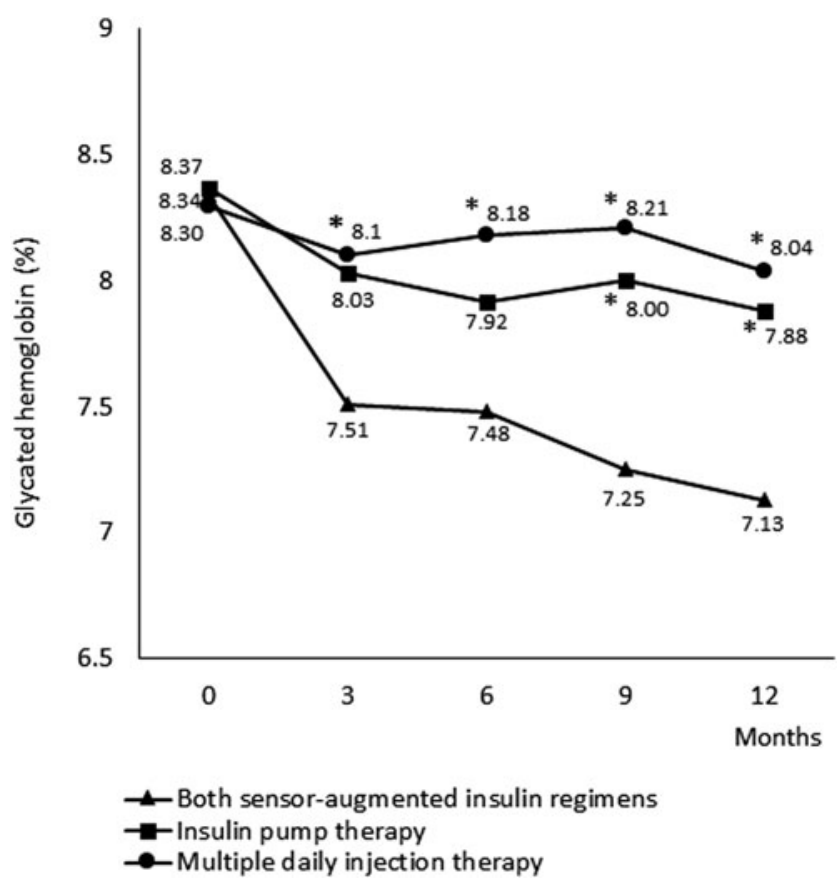

FIG. 1. Comparison of different treatment strategies for patient with type 1 diabetes during 1 year of follow-up: effect on $\mathrm{HbA}_{1 \mathrm{c}}$. Mean glycated hemoglobin levels (\%) at baseline and $3,6,9$, and 12 months in all groups. Asterisks denote $P<0.05$ for comparison between the sensor-augmented regimens group and the two SMBG groups (insulin pump and MDI therapy) at each time point. MDIs, multiple daily injections; SMBG, self-monitoring of blood glucose.

\section{Primary and secondary endpoints}

After a year, the SAIR group of patients had significantly lower $\mathrm{HbA}_{1 \mathrm{c}}(8.3 \% \pm 0.9 \%$ vs. $7.1 \% \pm 0.8 \%$ [67.5 $\pm 10.4 \mathrm{mmol} /$ mol vs. $54.5 \pm 9.1 \mathrm{mmol} / \mathrm{mol}] ; P<0.0001$ ) (Fig. 1). This improvement in $\mathrm{HbA}_{1 \mathrm{c}}$ was observed both in the subgroup with SAP $(8.2 \% \pm 0.9 \%$ vs. $7.1 \% \pm 0.9 \% \quad[66 \pm 9 \mathrm{mmol} / \mathrm{mol}$ vs. $53.9 \pm 10 \mathrm{mmol} / \mathrm{mol}] ; P=0.0025)$ and with MDIs + RT-CGM $(8.5 \% \pm 1.1 \%$ vs. $7.2 \% \pm 0.8 \%[69.3 \pm 12 \mathrm{mmol} / \mathrm{mol}$ vs. $55.3 \pm$ $8.7 \mathrm{mmol} / \mathrm{mol}] ; P=0.0034)$ compared with the study baseline (Fig. 2).

CSII alone also led to significant reduction of $\mathrm{HbA}_{1 \mathrm{c}}$ $(8.4 \% \pm 0.9 \%$ vs. $7.9 \% \pm 0.7 \%[68.3 \pm 9 \mathrm{mmol} / \mathrm{mol}$ vs. $62.7 \pm$ $8 \mathrm{mmol} / \mathrm{mol}$ ]; $P=0.048$ ), while in the group just on MDIs, no significant decrease of $\mathrm{HbA}_{1 \mathrm{c}}$ was observed $(8.3 \% \pm 0.8 \%$ vs. $8.0 \% \pm 0.9 \% \quad[67.2 \pm 9 \mathrm{mmol} / \mathrm{mol}$ vs. $64.4 \pm 10 \mathrm{mmol} / \mathrm{mol}]$; $P=0.40)$ (Fig. 1).

At 1 year, the mean difference in $\mathrm{HbA}_{1 \mathrm{c}}$ between the SAIR group and the MDI group was $-0.91 \%(-9.81 \mathrm{mmol} / \mathrm{mol})$ (95\% confidence interval [CI], $-1.47 \%$ to $-0.35 \%$ [ -15.96 to $-3.67 \mathrm{mmol} / \mathrm{mol}] ; P=0.002)$. Moreover, both SAIR strategies were superior to CSII; the mean difference was $-0.75 \%$ $(-8.11 \mathrm{mmol} / \mathrm{mol})(95 \% \mathrm{CI},-1.23 \%$ to $-0.26 \%[-13.41$ to $-2.81 \mathrm{mmol} / \mathrm{mol}$ ]; $P=0.0032$ ). The difference in $\mathrm{HbA}_{1 \mathrm{c}}$ between the SAIR group and the MDI group was significant from the third month and the difference between the SAIR group and the CSII group was significant from the ninth month (Fig. 1).

Importantly, superiority of both SAIRs in comparison with CSII only was not observed just for the SAP version of SAIR 
insulin pump therapy (without real-time CGM)

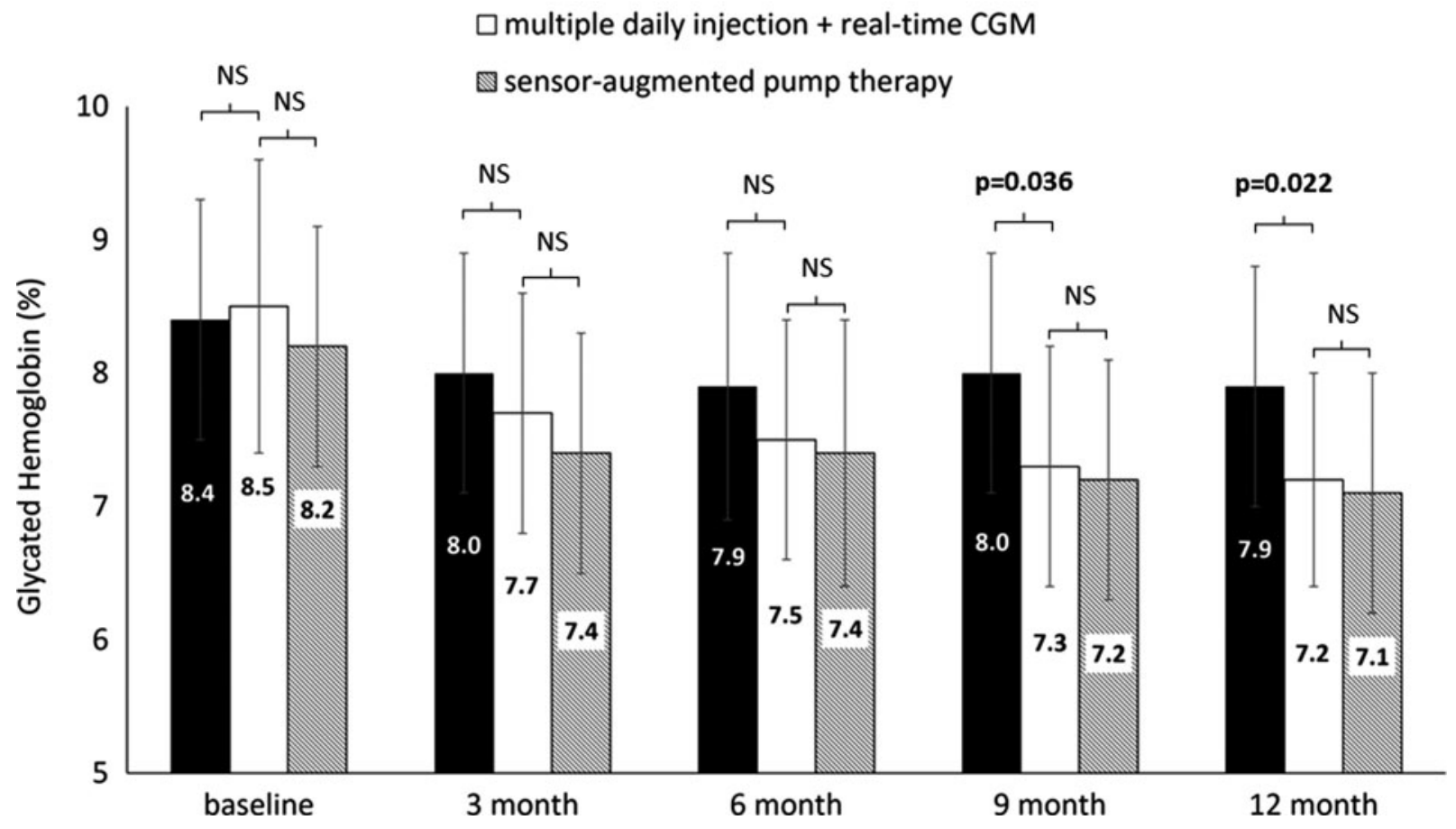

FIG. 2. Comparison of the effect on $\mathrm{HbA}_{1 \mathrm{c}}$ in patients with $\mathrm{MDIs}+$ real-time CGM therapy with sensor-augmented pump therapy and insulin pump therapy during the 52-week follow-up study. Mean glycated hemoglobin levels (\%) at baseline and 3, 6,9 , and 12 months. $P$ values $<0.05$ are statistically significant. CGM, continuous glucose monitoring; NS, not significant.

but also for the MDI version of SAIR for a between-group difference favoring the MDI + RT-CGM subgroup of $-0.66 \%$ $(-7.4 \mathrm{mmol} / \mathrm{mol})(95 \% \mathrm{CI},-1.23 \%$ to $-0.10 \%[-13.64$ to $-1.6 \mathrm{mmol} / \mathrm{mol}] ; P=0.022$ ). The difference in $\mathrm{HbA}_{1 \mathrm{c}}$ between CSII and MDI + RT-CGM groups started to be significant from the ninth month of this study (Fig. 2).

At the baseline, no patient met the ADA/ESDA goal for $\mathrm{HbA}_{1 \mathrm{c}}(<7.0 \%$ [53 mmol/mol] $)$, while at the end of this trial, $48 \%$ of subjects in the SAIR group (eight patients in SAP and five patients in MDI subgroups), $16 \%(n=3)$ of patients in the CSII group, and $18 \%(n=3)$ of individuals on MDIs achieved the $\mathrm{HbA}_{1 \mathrm{c}}$ target.

At 1 year, the average daily glucose level, as measured by RT-CGM or professional CGM, was significantly lower, both in the SAIR group $(10.6 \pm 1.5 \mathrm{mmol} / \mathrm{L}$ vs. $8.7 \pm 1.4 \mathrm{mmol} / \mathrm{L}$; $P<0.001)$ and in the CSII group $(10.7 \pm 1.2 \mathrm{mmol} / \mathrm{L}$ vs. $9.8 \pm 1.1 \mathrm{mmol} / \mathrm{L} ; P=0.04)$. This improvement in average CGM glucose was accompanied by an increase in the time in range $(4.0-10.0 \mathrm{mmol} / \mathrm{L}) ; 50 \% \pm 11 \%$ versus $69 \% \pm 11 \%$; $P<0.0001$, for SAIR and $51 \% \pm 10 \%$ versus $59 \% \pm 11 \%$, $P=0.03$, for CSII.

Compared with the baseline, $\mathrm{GV}$ was lower in the groups on SAIR (SD $: 4.0 \pm 0.7 \mathrm{mmol} / \mathrm{L}$ vs. $3.0 \pm 0.5 \mathrm{mmol} / \mathrm{L} ; P<0.0001)$ and with $\mathrm{CSII}\left(\mathrm{SD}_{T} 3.9 \pm 0.6 \mathrm{mmol} / \mathrm{L}\right.$ vs. $3.4 \pm 0.6 \mathrm{mmol} / \mathrm{L}$; $P<0.05)$. Additionally, significant reduction of the time spent in hypoglycemia was observed only in patients with SAIR $(8 \% \pm 4 \%$ vs. $6 \% \pm 3 \% ; P<0.01)$. For patients just on MDIs, no significant change in $\mathrm{GV}\left(\mathrm{SD}_{T} 3.8 \pm 1.0 \mathrm{mmol} / \mathrm{L}\right.$ vs. $3.8 \pm$ $1.1 \mathrm{mmol} / \mathrm{L} ; P=0.93)$ and in hypoglycemia $(6 \% \pm 4 \%$ vs. $7 \% \pm 5 \% ; P=0.68)$ was observed.

No difference in $\mathrm{HbA}_{1 \mathrm{c}}(7.2 \% \pm 0.8 \%$ vs. $7.3 \% \pm 0.9 \%$ [54 $\pm 9 \mathrm{mmol} / \mathrm{mol}$ vs. $56 \pm 10 \mathrm{mmol} / \mathrm{mol}] ; P=0.87$ ), hypo- glycemia $(6 \% \pm 4 \%$ vs. $6 \% \pm 3 \% ; P=0.91)$, and $\mathrm{GV}\left(\mathrm{SD}_{T}\right.$ $2.9 \pm 0.5 \mathrm{mmol} / \mathrm{L}$ vs. $3.0 \pm 0.4 \mathrm{mmol} / \mathrm{L} ; P=0.67)$ was observed in patients with the two types of CGM systems (DexCom G4 and Enlite sensor).

\section{Adverse event}

Throughout the study, two severe episodes of hypoglycemia were reported, one in the CSII group and one in the MDI group. No severe hypoglycemia in the SAIR group was reported. There was no ketoacidosis or sensor insertion site infection requiring assistance during a year of follow-up.

\section{Discussion}

To the best of our knowledge, this is the first prospective, 1-year real-life study simultaneously comparing four different treatment strategies based on different combinations of insulin delivery and monitoring systems. The sensor-augmented pump therapy for A1C Reduction (STAR3) study provided only comparison between SAP and MDIs where RT-CGM was not used. ${ }^{6}$ Thus, it was not possible to determine the contribution of each component of the system on results. The SWITCH Study ${ }^{7}$ showed that addition of RT-CGM to already established CSII therapy led to an improvement of glycemic control, while removal of RT-CGM resulted in a loss of this benefit. This implies that RT-CGM plays an important role in CSII patients. However, no patients with MDI therapy were investigated. The Juvenile Diabetes Research Foundation (JDRF) CGM studies investigated patients both on MDIs and CSII therapy. The JDRF, however, did not report the subgroup analyses comparing patients on MDI therapy with those on CSII therapy. ${ }^{11,12}$ 
Our study showed significant glycemic benefits in using RT-CGM, which were comparable for patients either on CSII or MDI therapy. Moreover, the usage of RT-CGM resulted in a sustained decrease of $\mathrm{HbA}_{1 \mathrm{c}}$ with a concurrent reduction of time spent in hypoglycemia, which has not always been described. ${ }^{6}$

Recent data from the T1D Exchange Clinic Registry ${ }^{1}$ show that only $\sim 30 \%$ of registered adults meet the ADA/ESDA goal for $\mathrm{HbA}_{1 \mathrm{c}}$ of $<7.0 \%$ (53 $\left.\mathrm{mmol} / \mathrm{mol}\right)$. Given the inclusion criteria of our study, at the baseline, no patient met the ADA/ ESDA goal either. However, after a year, our study showed that almost half of subjects in the SAIR group met the target for $\mathrm{HbA}_{1 \mathrm{c}}$.

The $\mathrm{HbA}_{1 \mathrm{c}}$ decrease with SAIR in this study was accompanied by improvements in other secondary endpoints, including increased time in target range (4.0-10.0 mmol/L) and reduced GV. For describing GV in the present trial, we used total standard deviation $\left(\mathrm{SD}_{T}\right)$. It has been suggested that although $\mathrm{SD}_{T}$ has limitations, ${ }^{13}$ more complex parameters of $\mathrm{GV}$ usually provide no additional information, ${ }^{4}$ and thanks to its simplicity, it is easy to calculate $\mathrm{SD}_{T}$ as a component of routine diabetes management. ${ }^{14}$

In our study, treatment with CSII only also resulted in reduction of $\mathrm{HbA}_{1 \mathrm{c}}$ and $\mathrm{GV}$, while in the group just on MDIs, significant decrease of $\mathrm{HbA}_{1 \mathrm{c}}$ and other endpoints was not achieved. One important result was that a combination of RTCGM and MDIs was clearly superior to the improvement with CSII therapy, comparable with the superiority of SAP over CSII only. This is an important result because long-term studies comparing CSII without CGM and MDIs + RT-CGM are lacking.

In the 6-month follow-up study performed by Garg et al., RT-CGM provided similar benefits in glucose control for patients using either MDIs or SAP. However, in contrast with our trial, the significant decrease in $\mathrm{HbA}_{1}$ was not seen, either in patients with MDIs + RT-CGM or SAP therapy. On the other hand, like numerous other trials, ${ }^{6,11,12}$ the study was performed with the older generation of CGM. Since that time, experience with CGM and particularly the accuracy and usability of current systems have substantially improved, ${ }^{15}$ which should translate into the results of newer studies. In a smaller study published by Tumminia et al., 14 patients (eight in the MDI + RT-CGM group and six in the SAP group) using RT-CGM $>40 \%$ of the time significantly had decreased $\mathrm{HbA}_{1 \mathrm{c}}$ after 6 months. This effect was more evident in the MDI + RT-CGM group than the SAP group. ${ }^{16}$

In comparison with some other studies, ${ }^{6,11,16}$ we observed higher adherence to the use of RT-CGM. This is important because sufficient sensor use is crucial to the success of CGM. ${ }^{6,7,11,17}$ In the present study, $100 \%$ of participants in the SAIR group wore a sensor for more than $70 \%$ of the required time. This good adherence in using RT-CGM can be explained by the fact that patients were actively consulted about the treatment modality that best met their needs. In our study, we saw a greater frequency of boluses in the SAIR group compared with both SMBG groups. However, the higher number of boluses in the SAIR group does not correspond with the substantially lower frequency of finger sticks performed per day (6.8 boluses/day vs. 3.7 finger sticks/day). This is despite the fact that patients were encouraged to perform confirmatory finger sticks before each treatment decision. They often did not comply with this advice. This was apparent especially in patients with Dexcom G4 sensor. One possible explanation is that with the improved accuracy of the newer generation of CGM systems, ${ }^{15}$ patients have more trust in CGM technology and sometimes provide the insulin dose adjustment without SMBG (although this procedure cannot yet be recommended for the systems used in this study).

Thus, more experience, improved accuracy, and usability of current CGM systems, high adherence to RT-CGM use, and patient's confidence in RT-CGM-all these aspects could be factors in our results.

There are also limitations. This was a nonrandomized study. Thus, although baseline $\mathrm{HbA}_{1 \mathrm{c}}$ was similar, the more motivated patients might have selected the insulin pumps and/or CGM. Another possible limitation is the different types of insulin pumps and CGM systems used in this study. However, this reflects real-life and day-to-day clinical practice. Moreover, the study is designed as a long-term followup and it is still ongoing after the first year. Thus, if we had not paid attention to patients' needs and randomized them, we would have expected a higher dropout and gradual loss of the ability to describe differences between study groups.

We believe that our findings could facilitate further discussion and possibly have an influence on diabetes care. Despite the potential benefits of using CSII therapy, with or without $\mathrm{CGM},{ }^{7,18}$ many patients still report barriers to using it. $^{19,20}$ Some of these patients might be willing to use and benefit from another advanced technology-RT-CGMwhere insulin is administered by MDIs.

In conclusion, in patients with type 1 diabetes with suboptimal glycemic control, both SAIRs, that is, SAP and MDIs + RT-CGM, were superior to MDIs or CSII therapy in reducing $\mathrm{HbA}_{1 \mathrm{c}}$, hypoglycemia, and the other endpoints. Both SAIRs provided comparable glycemic benefits. Hence, a combination of real-time CGM and MDIs can be considered as an equivalent alternative to SAP therapy for patients who are not willing to or cannot use insulin pumps.

\section{Acknowledgments}

This study was initiated, designed, and performed by the investigators and supported by the Grant 15-26705A of the Agency for Healthcare Research (AZV) of the Czech Republic.

\section{Author Disclosure Statement}

No competing financial interests exist.

\section{References}

1. Miller KM, Foster NC, Beck RW, et al.: T1D Exchange Clinic Network. Current state of type 1 diabetes treatment in the U.S.: updated data from the T1D Exchange clinic registry. Diabetes Care 2015;38:971-978.

2. Nathan DM, Cleary PA, Backlund JY, et al.; Diabetes Control and Complications Trial/Epidemiology of Diabetes Interventions and Complications (DCCT/EDIC) Study Research Group: Intensive diabetes treatment and cardiovascular disease in patients with type 1 diabetes. N Engl J Med 2005;353:2643-2653.

3. Diabetes Control and Complications Trial Research Group. The effect of intensive treatment of diabetes on the development and 
progression of long-term complications in insulin-dependent diabetes mellitus. N Engl J Med 1993;329:977-998.

4. Šoupal J, Škrha J Jr, Fajmon M, et al.: Glycemic variability is higher in type 1 diabetes patients with microvascular complications irrespective of glycemic control. Diabetes Technol Ther 2014;16:198-203.

5. Garg SK, Voelmle MK, Beatson CR, et al.: Use of continuous glucose monitoring in subjects with type 1 diabetes on multiple daily injections versus continuous subcutaneous insulin infusion therapy: a prospective 6-month study. Diabetes Care 2011;34:574-579.

6. Bergenstal RM, Tamborlane WV, Ahmann A, et al.; STAR 3 Study Group. Effectiveness of sensor-augmented insulinpump therapy in type 1 diabetes. N Engl J Med 2010;363: 311-320.

7. Battelino T, Conget I, Olsen B, et al.; SWITCH Study Group. The use and efficacy of continuous glucose monitoring in type 1 diabetes treated with insulin pump therapy: a randomised controlled trial. Diabetologia 2012;55:31553162.

8. Battelino T, Phillip M, Bratina N, et al.: Effect of continuous glucose monitoring on hypoglycemia in type 1 diabetes. Diabetes Care 34:795-800.

9. Garg SK, Schwartz S, Edelman SV: Improved glucose excursions using an implantable real-time continuous glucose sensor in adults with type 1 diabetes. Diabetes Care 2004;27:734-738.

10. Rodbard D, Jovanovic L, Garg S: Responses to continuous glucose monitoring in patients with type 1 diabetes using multiple daily injections and insulin pumps. Diabetes Technol Ther 2009;11:757-765.

11. Tamborlane WV, Beck RW, Bode BW, et al.; Juvenile Diabetes Research Foundation Continuous Glucose Monitoring Study Group. Continuous glucose monitoring and intensive treatment of type 1 diabetes. N Engl J Med 2008; 359:1464-1476.

12. Juvenile Diabetes Research Foundation Continuous Glucose Monitoring Study Group: Effectiveness of continuous glucose monitoring in a clinical care environment: evidence from the Juvenile Diabetes Research Foundation continuous glucose monitoring (JDRF-CGM) trial. Diabetes Care 2010;33:17-22.
13. Rodbard D: Interpretation of continuous glucose monitoring data: glycemic variability and quality of glycemic control. Diabetes Technol Ther 2009;11(Suppl 1):S-55-S-67.

14. Suh S, Kim JH: Glycemic variability: how do we measure it and why is it important? Diabetes Metab J 2015;39:273-282.

15. Rodbard D: Continuous glucose monitoring: a review of successes, challenges, and opportunities. Diabetes Technol Ther 2016;18 Suppl 2:S2-3-S2-13.

16. Tumminia A, Crimi S, Sciacca L, et al.: Efficacy of realtime continuous glucose monitoring on glycaemic control and glucose variability in type 1 diabetic patients treated with either insulin pumps or multiple insulin injection therapy: a randomized controlled crossover trial. Diabetes Metab Res Rev 2015;31:61-68.

17. Raccah D, Sulmont V, Reznik Y, et al.: Incremental value of continuous glucose monitoring when starting pump therapy in patients with poorly controlled type 1 diabetes: the RealTrend study. Diabetes Care 2009;32:2245-2250.

18. Steineck I, Cederholm J, Eliasson B, et al.; Swedish National Diabetes Register. Insulin pump therapy, multiple daily injections, and cardiovascular mortality in 18,168 people with type 1 diabetes: observational study. BMJ 2015; 350:h3234.

19. Liberman A, Buckingham B: Diabetes technology and the human factor. Diabetes Technol Ther 2016;18 Suppl 1: S101-S111.

20. Wong JC, Foster NC, Maahs DM, et al.; T1D Exchange Clinic Network. Real-time continuous glucose monitoring among participants in the T1D Exchange clinic registry. Diabetes Care 2014;37:2702-2709.

Address correspondence to: Jan Šoupal, $M D$ 3rd Department of Internal Medicine 1st Faculty of Medicine Charles University U Nemocnice 1 Prague 12808 Czech Republic

E-mail: jan.soupal@seznam.cz 